\section{Block JNK at the dock}

\section{By Michael J. Haas, Senior Writer}

A team of U.S. researchers has described a new mitogen-activated protein kinase 8 (JNK; MAPK8) inhibitor that targets JNK's substrate docking site-instead of its ATP binding site-to treat type 2 diabetes. ${ }^{1}$ The targeting strategy should be applicable to other diseases in which JNK is implicated and to the inhibition of other protein kinases.

The research team from the Burnham Institute for Medical Research, the University of California, San Diego and Invitrogen Corp.'s Invitrogen Discovery Services unit in Wisconsin was led by Maurizio Pellecchia, professor of infectious diseases at Burnham.

"Our goal is to provide chemical probes that provide information about the JNK docking site and its requirements," Pellecchia told SciBX. "This is a basic science approach with a clear translational component. The compounds themselves are free for research purposes."

\section{JNK bonds}

The three c-Jun N-terminal kinases (JNK1; JNK2; JNK3) are members of the mitogen-activated protein kinase (MAPK) family and are activated by cytokines or cellular stress. JNK and many of its substrates, including c-Jun, activating transcription factor 2 (ATF2) and insulin receptor substrate 1 (IRS1), have been implicated in the pathogenesis of diabetes and other metabolic disorders, as well as in cancer and inflammatory, vascular and neurodegenerative diseases. ${ }^{2}$

According to Pellecchia, studies of JNK's role in disease have used either JNK knockout models or pharmacological inhibitors of JNK.

"Most JNK inhibition studies have focused on using ATP mimetics" because ATP-kinase interactions are more readily characterized than substrate-kinase interactions, and ATP is readily mimicked by many other small molecules, he said. "The problem is that the ATP binding site is highly conserved across all protein kinases," posing a significant challenge to selective targeting of JNK.

"Even SP600125, the gold standard of JNK inhibition targeting the ATP binding site, inhibits many other kinases," Pellecchia noted.

A few other JNK inhibition studies have focused on JNK's substrate docking site. JNK activation is facilitated by mitogen-activated protein kinase 8 interacting protein 1 (JIP1; MAPK8IP1; IB1), which binds simultaneously to JNK's substrate docking site and to other kinases responsible for JNK phosphorylation.
Studies over the past decade have shown that two peptide segments of JIP1's JNK-binding domain are potent inhibitors of JNK. ${ }^{3,4}$ Conjugates of these peptide segments with cell-penetrating peptides have been investigated as therapeutics for type 2 diabetes, ${ }^{5,6}$ but development of the conjugates has been hindered by poor cell permeability, dosing limitations and short in vivo half-life.

In work published in the Proceedings of the National Academy of Sciences, Pellecchia's team set out to study the substrate docking site, which is identical in JNK1, JNK2 and JNK3, with the goal of identifying highly specific small molecule JNK inhibitors.

First, the team screened a library of about 30,000 compounds from two commercially available libraries-one from Maybridge Corp. and the other from ASDI BioSciences-to look for small molecules that disrupted the interaction between JNK1 and pepJIP1, one of the JIP1 peptide segments known to inhibit JNK.

They subjected the hits from this screen to a second round of screening, using Invitrogen's LanthaScreen kinase assay to select for compounds that inhibited JNK1's phosphorylation of ATF2. Resulting hits underwent a final round of screening to rule out overly cytotoxic compounds. The result was lead molecule BI$78 \mathrm{D} 3$.

Subsequent computational modeling and NMR studies showed that BI-78D3 bound JNK1's substrate docking site in a manner similar to that observed in the crystal structure of the JNK1-pepJIP1 complex, which was solved by researchers at CrystalGenomics Inc. in $2004 .{ }^{4}$

Next, the team showed that in human cells, BI-78D3 prevented JNK phosphorylation of c-Jun without affecting p38 MAPK phosphorylation of ATF2 in the same cells. p38 is also activated by cytokines and cellular stress and is structurally similar to JNK.

Lastly, BI-78D3 restored insulin sensitivity in a mouse model of type 2 diabetes.

\section{c-Jun-worthy vessels}

The team wrote in PNAS that their results demonstrated that BI-78D3 was a potential new treatment for diabetes, and that blocking a kinase's substrate docking site, rather than its ATP binding site, could be a new strategy for selective targeting of protein kinases.

Teo Uysal, president and CEO of Syndexa Pharmaceuticals Corp., said BI-78D3 "appeared to be JNK-selective-at least compared to p38 MAPK - and showed the desired activity in cell- and animal-based assays." He also noted that findings were consistent with Syndexa's work that established a role for chronic JNK activation in diabetes, obesity and low-grade inflammatory responses.

Syndexa has small molecule programs targeting JNK and endoplasmic reticulum stress in preclinical development to treat metabolic diseases. The company hopes to take a compound into clinical testing to treat type 2 diabetes by $2011 .^{7}$

Uysal added that targeting the substrate docking site avoids another issue-besides selectivity - associated with targeting the ATP binding 
site. "ATP concentrations inside cells reach millimolar levels," he said. Inhibition of JNK at this site therefore "requires a compound to be very potent in displacing and competing against ATP for binding."

Christophe Bonny, president and CSO of Xigen S.A., voiced similar views. "The specificity of inhibitors targeting ATP binding sites is terrible," he said.

In 2006, Bonny and researchers at University Hospital Lausanne (CHUV) examined the activity of two inhibitors that targeted JNK's ATP binding site against about 300 other kinases. They found that the two inhibitors blocked $30-40 \%$ of those other kinases at least as well as they blocked JNK. ${ }^{8}$

Thus, ATP binding site inhibitors "have incredibly high side effects due to the nonspecific targeting of other kinases," Bonny said.

Therefore, and before deciding the compound is truly a selective JNK inhibitor, Bonny wants to see BI-78D3's inhibition of JNK compared to kinases besides p38 MAPK.

Taking the issue a step further, Seonggu Ro, CTO of CrystalGenomics, said that although the cell- and animal-based studies described in PNAS supported the conclusion that BI-78D3 was a selective JNK inhibitor, it would be important "to obtain direct evidence for BI-78D3's binding to the JIP1 binding site of JNK1 through X-ray crystallography."

Besides confirming the specific interaction of BI-78D3 with JNK, the information provided by X-ray crystal structures would be "highly useful for the discovery of new therapeutics that exploit the inhibitory mechanism," Ro said.

Pellecchia said it was not necessary to demonstrate BI-78D3's selectivity for JNK beyond comparing it to p38 MAPK. "p38 is the standard comparison because it is so closely related to JNK," he said. "So if you don't inhibit this, it is hard to believe that you would inhibit any other kinases."

He added that his team has shown that the compound does not inhibit protein kinase B (PKB; Akt) or phosphoinositide 3-kinase (PI3K), but did not report those results in the PNAS paper.

Xigen's lead compound is XG-102, a selective JNK inhibitor composed of D-JNK-1 conjugated to a cell-penetrating HIV-TAT peptide. D-JNK-1 is a complete D-amino-acid analog of a 21 -amino-acid sequence of JIP1's binding domain.

Xigen and partner Auris Medical AG are running a Phase II trial of XG-102 to treat acute acoustic trauma and hearing loss resulting from inflammation of the inner ear. Xigen is also completing a Phase I trial of the compound to treat stroke and plans to start Phase II trials to treat stroke and inflammatory indications in 2009.

Bonny and researchers at CHUV had previously described the potential of the D-JNK-1-HIV-TAT conjugate as a treatment for dia- betes, ${ }^{5}$ but discontinued their research because the dose required to have an effect in animal models was too high, in the $\mathrm{mg} / \mathrm{kg}$ range. By comparison, XG-102 "showed good effect" at doses of $1 \mu \mathrm{g} / \mathrm{kg}$ in animal models of stroke, according to Bonny.

CrystalGenomics utilizes kinase-specific compound libraries, generated with the company's structural chemoproteomics technology, to identify molecules that target disease-related kinases. The company's lead compound, an inhibitor of glycogen dependent kinase 3 (GSK3), is in IND-enabling studies to treat diabetes, cancer and Alzheimer's disease $(\mathrm{AD})$.

\section{Double docking}

Besides continuing to study the role of the JNK docking site in diabetes and other indications such as melanoma, stroke and asthma, Pellecchia said the Burnham team has been researching bifunctional JNK inhibitors-molecules capable of blocking both the ATP binding site and substrate docking site simultaneously. The team published proof of concept for such bifunctional molecules earlier this year. ${ }^{9}$

"We have now designed bifunctional JNK inhibitors with singledigit nanomolar affinity for JNK," which will be described in a forthcoming publication, he said.

Burnham holds an international patent on the methods and findings reported in PNAS.

Haas, M.J. SciBX 1(39); doi:10.1038/scibx.2008.940

Published online Oct. 30, 2008

\section{REFERENCES}

1. Stebbins, J. et al. Proc. Natl. Acad. Sci. USA; published online Oct. 15, 2008; doi:10.1073/pnas.0805677105

Contact: Maurizio Pellecchia, Burnham Institute for Medical Research, La Jolla, Calif.

e-mail: mpellecchia@burnham.org

2. Manning, A. \& Davis, R. Nat. Rev. Drug Discov. 2, 554-565 (2003)

3. Barr, R. et al. J. Biol. Chem. 277, 10987-10997 (2002)

4. Heo, Y. et al. EMBO J. 23, 2185-2195 (2004)

5. Bonny, C. et al. Diabetes 50, 77-82 (2001)

6. Kaneto, H. et al. Nat. Med. 10, 1128-1132 (2004)

7. Flanagan, M. BioCentury 16(2), 31; Jan. 7, 2008

8. Guenat, S. et al. J. Biomol. Screen 11, 1015-1026 (2006)

9. Vazquez, J. et al. J. Med. Chem. 51, 3460-3465 (2008)

\section{COMPANIES AND INSTITUTIONS MENTIONED}

ASDI BioSciences, Newark, Del.

Auris Medical AG, Basel, Switzerland

Burnham Institute for Medical Research, La Jolla, Calif.

CrystalGenomics Inc. (KOSDAQ:083790), Daejon, South Korea

Invitrogen Corp. (NASDAQ:IVGN), Carlsbad, Calif.

Maybridge Corp., Cornwell, U.K.

Syndexa Pharmaceuticals Corp., Cambridge, Mass.

University Hospital Lausanne, Lausanne, Switzerland

University of California, San Diego, La Jolla, Calif.

Xigen S.A., Lausanne, Switzerland 